\title{
Some Results of Upper and Lower $M$-Asymmetric Irresolute Multifunctions in Bitopological Spaces
}

\author{
Levy K. Matindih ${ }^{*}{ }^{\mathbb{D}}$, Edwin Moyo ${ }^{1}$, Davy K. Manyika', Timothy Sinyangwe ${ }^{2}$ \\ ${ }^{1}$ Department of Science \& Mathematics, Mulungushi University, Kabwe, Zambia \\ ${ }^{2}$ Department of Mathematics, Copperbelt University, Kitwe, Zambia \\ Email: ^kahyamatindih99@gmail.com
}

How to cite this paper: Matindih, L.K. Moyo, E., Manyika, D.K. and Sinyangwe, T. (2021) Some Results of Upper and Lower $M$-Asymmetric Irresolute Multifunctions in Bitopological Spaces. Advances in Pure Mathematics, 11, 611-627.

https://doi.org/10.4236/apm.2021.116040

Received: May 5, 2021

Accepted: June 27, 2021

Published: June 30, 2021

Copyright $\odot 2021$ by author(s) and Scientific Research Publishing Inc. This work is licensed under the Creative Commons Attribution International License (CC BY 4.0).

http://creativecommons.org/licenses/by/4.0/

\begin{abstract}
In this paper, we aim to introduce and study some basic properties of upper and lower $M$-asymmetric irresolute multifunctions defined between asymmetric sets in the realm of bitopological spaces with certain minimal structures as a generalization of irresolute functions deal to Crossley and Hildebrand [1] and upper and lower irresolute Multifunctions deal to Popa [2].
\end{abstract}

\section{Keywords}

$\mathscr{T}_{i} \cdot T_{j}$-Semiopen Sets, $M$-Space, $M$-Asymmetric Semiopen and Semiclosed

Sets, Upper (Lower) Irresolute Multifunctions and Upper (Lower)

$M$-Asymmetric Irresolute Multifunctions

\section{Introduction}

Topology and its vital role to continuity have received considerable attention by several authors not only in the field of functional analysis but also in other branches of applied science. Continuity and multifunctions, the basic concepts in the theory of classical point set topology which plays an important role not only in the field of functional analysis but also in applied sciences: mathematical economics, control theory, and fuzzy topology have also received considerable attention by many scholars. In this regard, several scholars have generalizations these notions of continuity to (bi-)topological spaces using the weaker forms of open and closed sets the semiopen and semiclosed sets see: [1] [3] [4] [5] [6] [7].

The fundamental idea of semiopen sets and semi-continuous functions in topological spaces was first introduced by Levine [3] and these concepts have been extended to the realm of bitopological spaces by Maheshwari and Prasad [4], 
and also Bose [8]. Berge [9] on the other hand introduced the notion of upper and lower continuous multifunctions and lately, Popa [5] generalized this concept to the settings of bitopological spaces in which he studied how the conserving properties of connectedness, compactness and paracompactness are preserved by multifunctions between bitopological spaces. In 2000, Noiri and Popa [10] introduced and studied the notion of upper and lower $M$-continuous multifunctions as a generalization of upper (lower) continuous multifunction and $M$-continuous function deal to Berge [9] and, Popa and Noiri [7] respectively. They showed that the upper (lower) continuity of multifunctions has properties similar to those of upper (lower) continuous functions and continuous multifunctions between topological spaces. Recently, Matindih and Moyo [11] have extended the ideas in [10] and studied $M$-asymmetric semiopen sets and semicontinuous multifunctions from which they observed that, such functions have properties similar to those of upper (lower) continuous functions and $M$-continuous multifunctions between topological spaces, with the only difference that, the semiopen sets in use belonged to two topologies.

In 1972, the notion of irresolute functions was introduced and their fundamental properties were investigated by Crossley and Hildebrand [1]. They discovered that, most irresolute functions are not necessarily continuous and neither are continuous functions necessarily irresolute. As an extension and generalization of this idea, Ewert and Lipski [12], then studied the concept of upper and lower irresolute multivalued mappings and, followed by Popa [2] who looked at some characterizations of upper and lower irresolute multifunctions in topological spaces.

In this present paper, we introduce and study some basic properties of $M$-asymmetric irresolute multifunctions defined between sets satisfying certain minimal conditions in the framework of bitopological spaces, as a generalization of results deal to Crossley and Hildebrand [1], Popa [2] and, Noiri and Popa [10].

The organization of this paper is as follows. Section 2 presents some necessary preliminaries concerning semiopen sets, $m$-asymmetric semiopen sets and $M$-(asymmetric semi)-continuous multifunctions [11]. In section 3, we present and discuss some results of $M$-asymmetric irresolute multifunctions as a generalized idea for irresolute functions [1] and upper and lower $M$-continuous multifunctions [10]. Section 4 gives some concluding remarks.

\section{Preliminaries and Basic Properties}

This section presents some important properties and notations to be used in this paper. For more details, we refer the reader to ([2] [4] [5] [6] [8] [10] [11] [13]).

By a bitopological space $\left(X, \mathscr{T}_{1}, \mathscr{T}_{2}\right)$, first introduced by Kelly ([13]), we mean a nonempty set $X$ on which are defined the left and right topologies $\mathscr{T}_{1}$ and $\mathscr{T}_{2}$. In what follows, $\left(X, \widetilde{T}_{1}, \mathscr{T}_{2}\right)$ or in short $X$ will denote a bitopological space unless clearly stated. For a bitopological space $\left(X, \widetilde{T}_{i}, \mathscr{T}_{j}\right), i, j=1,2$; 
$i \neq j$, we shall denote the interior and closure of a subset $A$ of $X$ with respect to the topology $\widetilde{T_{i}}=\mathscr{T}_{j}$ by $\operatorname{Int}_{\widetilde{T_{i}}}(A)$ and $C l_{\widetilde{J_{j}}}(A)$ respectively.

Definition 2.1. [8] [13] Let $\left(X, \mathscr{T}_{i}, \mathscr{T}_{j}\right), i, j=1,2 ; i \neq j$ be a bitopological space and let $\mathrm{A}$ be any nonvoid subset of $\mathrm{X}$.

1) $A$ is said to be $\mathscr{T}_{i} \mathscr{T}_{j}$-open if $A \in \mathscr{T}_{i} \cup \mathscr{T}_{j}$; i.e., $A=A_{i} \cup A_{j}$ where $A_{i} \in \mathscr{T}_{i}$ and $A_{j} \in \mathscr{T}_{j}$. The complement of an $\mathscr{T}_{i} \mathscr{T}_{j}$-open set is a $\mathscr{T}_{i} \mathscr{T}_{j}$-closed set.

2) The $\mathscr{T}_{i} \mathscr{T}_{j}$-interior of $A$ denoted by $\operatorname{Int}_{\overparen{\widehat{T}}}\left(\operatorname{Int}_{\overparen{\sqrt{j}}}(A)\right)$ (or $\left.\mathscr{T}_{i} \cdot \mathscr{T}_{j} \operatorname{Int}(A)\right)$ is the union of all $\widetilde{T}_{i} \cdot T_{j}$-open subsets of $X$ contained in $A$. Evidently, provided $A=\operatorname{Int}_{\overparen{T_{i}}}\left(\operatorname{Int} t_{\widetilde{\gamma_{j}}}(A)\right)$, then $A$ is $\mathscr{T}_{i} \mathscr{T}_{j}$-open.

3) The $\mathscr{T}_{i} \mathscr{T}_{j}$-closure of $A$ denoted by $C l_{\widetilde{S}_{i}}\left(C l_{\widehat{J}_{j}}(A)\right)$ is defined to be the intersection of all $\widetilde{T}_{i} T_{j}$-closed subsets of $X$ containing $A$. Note that asymme-

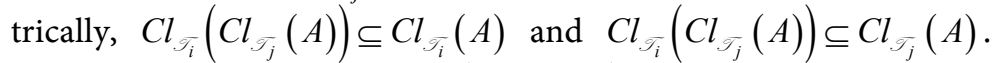

Definition 2.2. [4] [8] Let $\left(X, \mathscr{T}_{i}, \mathscr{T}_{j}\right), i, j=1,2 ; i \neq j$ be a bitopological space and, let $\mathrm{A}$ and $\mathrm{B}$ be non-void subsets of $\mathrm{X}$.

1) $A$ is said to be $\mathscr{T}_{i} \cdot T_{j}$-semiopen in $X$ provided there is a $\mathscr{T}_{i}$-open subset $O$ of $X$ such that $O \subseteq A \subseteq C l_{\overparen{\gamma_{j}}}(O)$, equivalently $A \subseteq C l_{\widetilde{\gamma_{j}}}\left(\operatorname{Int}_{\widetilde{\tau_{i}}}(A)\right)$. It's complement is said to be $\mathscr{T}_{i} \mathscr{T}_{j}$-semiclosed.

2) The $\mathscr{T}_{i} \cdot \mathscr{T}_{j}$-semiinterior of $A$ denoted by $\mathscr{T}_{i} \mathscr{T}_{j} \operatorname{sint}(A)$ is defined as the union of $\mathscr{T}_{i} \mathscr{T}_{j}$-semiopen subsets of $X$ contained in $A$. The $\mathscr{T}_{i} \mathscr{T}_{j}$-semiclosure of $A$ denoted by $\widetilde{T}_{i} \widetilde{T}_{j}-s C l(A)$, is the intersection of all $\widetilde{T}_{i} \cdot \widetilde{T}_{j}$-semiclosed sets of $X$ containing $A$.

3) $B$ is said to be a $\mathscr{T}_{i} \mathscr{T}_{j}$-semi-neighbourhood of $x \in X$ provided there is a $\mathscr{T}_{i} \mathscr{T}_{j}$-semiopen subset $O$ of $X$ such that $x \in O \subseteq B$.

The family of all $\mathscr{T}_{i} \mathscr{T}_{j}$-semiopen and $\mathscr{T}_{i} \mathscr{T}_{j}$-semiclosed subsets of $X$ are denote by $\mathscr{T}_{i} \mathscr{T}_{j} S O(X)$ and $\mathscr{T}_{i} \mathscr{T}_{j} S C(X)$ respectively.

Definition 2.3. [6] [10] A subfamily $m_{X}$ of a power set $\mathscr{P}(X)$ of a nonevoid set $\mathrm{X}$ is said to be a minimal structure (briefly $\mathrm{m}$-structure) on $\mathrm{X}$ if both $\varnothing$ and $\mathrm{X}$ lies in $m_{X}$. The pair $\left(X, m_{X}\right)$ is called an $\mathrm{m}$-space and the members of $\left(X, m_{X}\right)$ is said to be $m_{X}$-open.

Definition 2.4. [11] Let $\left(X, \mathscr{T}_{i}, \mathscr{T}_{j}\right), i, j=1,2 ; i \neq j$ be a bitopological space and $m_{X}$ a minimal structure on $\mathrm{X}$ generated with respect to $m_{i}$ and $m_{j}$. An ordered pair $\left(\left(X, \mathscr{T}_{i}, \mathscr{T}_{j}\right), m_{X}\right)$ is called a minimal bitopological space.

Since the minimal structure $m_{X}$ is determined by the left and right minimal structures $m_{i}$ and $m_{j}$, generated by the two topologies $\mathscr{T}_{i}$ and $\mathscr{T}_{j}$, $i, j=1,2 ; i \neq j$, we shall denote it by $m_{i j}(X)$ (or simply $m_{i j}$ ) in the sense of Matindih and Moyo [11], and call the pair $\left(\left(X, \mathscr{T}_{i}, \mathscr{T}_{j}\right), m_{i j}\right)$ (or $\left.\left(X, m_{i j}\right)\right)$ a minimal bitopological space unless explicitly defined.

Definition 2.5. A minimal structure $m_{X}$ of a bitoplogical space $\left(X, \mathscr{T}_{i}, \mathscr{T}_{j}\right)$, $i, j=1,2 ; i \neq j$; is said to have property ( $\mathscr{B}$ ) of Maki [6] if the union of any collection of $m_{i j}(X)$-open subsets of $\mathrm{X}$ belongs to $m_{X}$.

Definition 2.6. [11] Let $\left(X, m_{i j}\right), i, j=1,2 ; i \neq j$ be a minimal bitopological space and A a subset of $\mathrm{X}$. A is said to be: 
1) $m_{i j}$-semiopen in $X$ if there exists an $m_{i}$-open set $O$ such that $O \subseteq A \subseteq C l_{m_{j}}(O)$ or equivalently, $A \subseteq C l_{m_{j}}\left(\operatorname{Int}_{m_{i}}(A)\right)$.

2) $m_{i j}$-closed in $X$ if there exists an $m_{i}$-open set $O$ such that $C l_{m_{j}}(A) \subseteq O$ whenever $A \subseteq O$ or equivalently $\operatorname{Int}_{m_{j}}\left(C l_{m_{i}}(A)\right) \subseteq A$.

We shall denote the collection of all $m_{i j}$-semiopen and $m_{i j}$-semiclosed sets in $\left(X, m_{i j}\right)$ by $m_{i j} s O(X)$ and $m_{i j} s C(X)$ respectively.

Remark 2.7. [11] Let $\left(X, m_{i j}\right), i, j=1,2 ; i \neq j$ be a minimal bitopological space.

1) if $m_{i}=\mathscr{T}_{i}$ and $m_{j}=\mathscr{T}_{j}$, the any $m_{i j}$-semiopen set is $\mathscr{T}_{i} \mathscr{T}_{j}$-semiopen.

2) every $m_{i j}$-open (resp. $m_{i j}$-closed) set is $m_{i j}$-semiopen (resp. $m_{i j}$-semiclosed), but the converse is not generally true, see Examples 3.5 [11].

The $m_{i j}$-open sets and the $m_{i j}$-semiopen sets are not stable for the union. However, for certain $m_{i j}$-structures, the class of $m_{i j}$-semiopen sets are stable under union of sets, as the Lemma below clarifies.

Lemma 2.8. [11] Let $\left(X, m_{i j}\right), i, j=1,2 ; i \neq j$ be an $m_{i j}$-space and $\left\{A_{\gamma}: \gamma \in \Gamma\right\}$ be a family of subsets of $X$. Then, the properties below hold:

1) $\bigcup_{\gamma \in \Gamma} A_{\gamma} \in m_{i j} s O(X)$ provided for all $\gamma \in \Gamma, A_{\gamma} \in m_{i j} s O(X)$.

2) $\bigcap_{\gamma \in \Gamma} A_{\gamma} \in m_{i j} s C(X)$ provided for all $\gamma \in \Gamma, A_{\gamma} \in m_{i j} s C(X)$.

Remark 2.9. [11] It should generally be noted that, the intersection of any two $m_{i j}$-semiopen sets may not be $m_{i j}$-semiopen in a minimal bitopological space $\left(X, m_{i j}\right)$, as illustrated in Example 3.9 [11].

Definition 2.10. [11] Let $\left(X, m_{i j}\right), i, j=1,2 ; i \neq j$ be an $m_{i j}$-space. A subset:

1) $O$ of $X$ is an $m_{i j}$-semineighborhood of a point $x$ of $X$ if there exists an $m_{i j}$-semiopen subset $U$ of $X$ such that $x \in U \subseteq O$.

2) $O$ of $X$ is an $m_{i j}$-semineighborhood of a subset $A$ of $X$ if there exists an $m_{i j}$-semiopen subset $U$ of $X$ such that $A \subseteq U \subseteq O$.

Definition 2.11. [11] Let $\left(X, m_{i j}\right), i, j=1,2 ; i \neq j$ be an $m_{i j}$-space and A a none-void subset of $\mathrm{X}$. Then, we denoted and defined the $m_{i j}$-semiinterior and $m_{i j}$-semiclosure of A respectively by:

1) $m_{i j} \operatorname{sint}(A)=\bigcup\left\{U: U \subseteq A\right.$ and $\left.U \in m_{i j} s O(X)\right\}$.

2) $m_{i j} s C l(A)=\bigcap\left\{F: A \subseteq F\right.$ and $\left.F \in m_{i j} s C(X)\right\}$.

Remark 2.12. [11] For any bitopological spaces $\left(X, \mathscr{T}_{1}, \mathscr{T}_{2}\right)$ :

1) $\mathscr{T}_{i} \mathscr{T}_{j} S O(X)$ is a minimal structures of $X$.

2) In the following, we denote by $m_{i j}(X)$ a minimal structure on $X$ as a generalization of $\mathscr{T}_{i}$ and $\mathscr{T}_{j}$. For a none-void subset $A$ of $X$, if $m_{i j}(X)=\mathscr{T}_{i} \cdot{ }_{j} s O(X)$, then by Definition 2.11 .

a) $m_{i j} \operatorname{Int}(A)=\mathscr{T}_{i} \cdot \mathscr{T}_{j} \operatorname{sint}(A)$,

b) $m_{i j} C l(A)=\mathscr{T}_{i} \mathscr{T}_{j} s C l(A)$.

Lemma 2.13. [11] For any $m_{i j}$-space $\left(X, m_{i j}\right), i, j=1,2 ; i \neq j$ and nonevoid subsets $A$ and $B$ of $X$, the following properties of $m_{i j}$-semiclosure and $m_{i j}$-semiinterior holds. 
1) $m_{i j} \operatorname{sint}(A) \subseteq A$ and $m_{i j} s C l(A) \supseteq A$.

2) $m_{i j} \operatorname{sint}(A) \subseteq m_{i j} \operatorname{sint}(B)$ and $m_{i j} s C l(A) \subseteq m_{i j} s C(B)$ provided $A \subseteq B$.

3) $m_{i j} \operatorname{sint}(\varnothing)=\varnothing, m_{i j} \operatorname{sint}(X)=X, m_{i j} s C l(\varnothing)=\varnothing$ and $m_{i j} s C l(X)=X$.

4) $A=m_{i j} \operatorname{Int}(A)$ provided $A \in m_{i j} S O(X)$.

5) $A=m_{i j} s C l(A)$ provided $X \backslash A \in m_{i j} s O(X)$.

6) $m_{i j} \operatorname{sint}\left(m_{i j} \operatorname{sint}(A)\right)=m_{i j} \operatorname{sint}(A)$ and $m_{i j} \operatorname{sl}\left(m_{i j} s C l(A)\right)=m_{i j} \operatorname{sCl}(A)$

Lemma 2.14. [11] Let $\left(X, m_{i j}\right), i, j=1,2 ; i \neq j$ be an $m_{i j}$-space and $A$ a none-void subset of $X$. For each $U \in m_{i j} S O(X)$ containing $x_{o}, U \cap A \neq \varnothing$ if and only if $x_{o} \in m_{i j} s C l(A)$.

Lemma 2.15. [11] For an $m_{i j}$-space $\left(X, m_{i j}\right), i, j=1,2 ; i \neq j$ and any none-void subset $A$ of $X$, the properties below holds.

1) $m_{i j} \operatorname{sCl}(X \backslash A)=X \backslash\left(m_{i j} \operatorname{sint}(A)\right)$,

2) $m_{i j} \operatorname{sint}(X \backslash A)=X \backslash\left(m_{i j} s C l(A)\right)$.

Lemma 2.16. [11] For an $m_{i j}$-space $\left(X, m_{i j}\right), i, j=1,2 ; i \neq j$ and any none-void subset $A$ of $X$, the properties below are true:

1) $m_{i j} s C l(A)=\operatorname{Int}_{m_{j}}\left(C l_{m_{i}}(A)\right) \cup A$.

2) $m_{i j} S C l(A)=\operatorname{Int}_{m_{j}}\left(C l_{m_{i}}(A)\right)$ provided $A \in m_{i j} O(X)$. The converse to this assertion is not necessarily true.

Example 2.17. [11] In this example, it is shown that, the converse to part (2) of Lemma 2.16 is not necessarily true: Define the two minimal structures $m_{1}$ and $m_{2}$ on $X=\{-3,-2,-1,0,1,2\}$ by $m_{1}=\{\varnothing,\{-2,1\}, X\}$ and $m_{2}=\{\varnothing,\{-3\},\{2\}, X\}$. Clearly, $\{-2,1\}$ is only $m_{i}$-open but not $m_{i j}$-open even thought $m_{i j} s C l(\{-2,1\})=\operatorname{Int}_{m_{j}}\left(C l_{m_{i}}(\{-2,1\})\right)$.

Remark 2.18. [11] For a bitopological space $\left(X, \mathscr{T}_{i}, \mathscr{T}_{j}\right), i, j=1,2 ; i \neq j$ the families $\mathscr{T}_{i} \mathscr{T}_{j} O(X)$ and $m_{i j} S O(X)$ are all $m_{i j}$-structures of $\mathrm{X}$ satisfying property $\mathscr{B}$.

Lemma 2.19. [11] For an $m_{i j}$-space $\left(X, m_{i j}\right), i, j=1,2 ; i \neq j$ with $m_{i j}$ satisfying property $(\mathscr{B})$ and subsets $A$ and $F$ of $X$, the properties below holds:

1) $m_{i j} \operatorname{sint}(A)=A$ provided $A \in m_{i j} S O(X)$.

2) If $X \backslash F \in m_{i j} S O(X)$, then $m_{i j} s C l(F)=F$.

Lemma 2.20. [11] For any $m_{i j}$-space $\left(X, m_{i j}\right), i, j=1,2 ; i \neq j$ with $m_{i j}$ satisfying property $\mathscr{B}$ and any none-void subset $A$ of $X$, the properties outlined below holds.

1) $A=m_{i j} \operatorname{sint}(A)$ if and only if $A$ is an $m_{i j}(X)$-semiopen set.

2) $A=m_{i j} s C l(A)$ if and only if $X \backslash A$ is an $m_{i j}$-semiopen set.

3) $m_{i j} \operatorname{sint}(A)$ is $m_{i j}$-semiopen.

4) $m_{i j} s C l(A)$ is $m_{i j}$-semiclosed.

Lemma 2.21. [11] Let $\left(X, m_{i j}\right), i, j=1,2 ; i \neq j$ be an $m_{i j}$-space with $m_{i j}$ satisfying the property $\mathscr{B}$ and let $\left\{A_{\gamma}: \gamma \in \Gamma\right\}$ be an arbitrary collection of subsets of $X$. Then, $\bigcup_{\gamma \in \Gamma} A_{\gamma} \in m_{i j} S O(X)$ provided $A_{\gamma} \in m_{i j} s O(X)$ for every $\gamma \in \Gamma$.

Lemma 2.22. [11] Let $\left(\left(X, \mathscr{T}_{i}, \mathscr{T}_{j}\right), m_{i j}\right), i, j=1,2 ; i \neq j$ be an $m_{i j}$-space and $A$ a non-void subset of $X$. Provided $m_{i j}$-satisfy property $\mathscr{B}$, then: 
1) $m_{i j} s C l(A)=A \cup \operatorname{Int}_{m_{i}}\left(C l_{m_{i}}(A)\right)$, and

2) $m_{i j} \operatorname{sint}(A)=A \cap C l_{m_{i}}\left(\operatorname{Int}_{m_{i}}(A)\right)$ holds.

If the property $\mathscr{B}$ of Make is removed in the previous Lemma, the equality does not necessarily hold, refer to Example 3.23 [11].

Lemma 2.23. [11] For a minimal bitopological space $\left(\left(X, \mathscr{T}_{i}, \mathscr{T}_{j}\right), m_{i j}(X)\right)$, $i, j=1,2 ; i \neq j$, and any subset $U$ of $X$, the properties below holds.

1) $m_{i j} \operatorname{sint}(U) \subseteq C l_{m_{j}}\left(\operatorname{Int}_{m_{i}}\left(m_{i j} \operatorname{sint}(U)\right)\right) \subseteq C l_{m_{j}}\left(\operatorname{Int}_{m_{i}}(U)\right)$.

2) $\operatorname{Int}_{m_{j}}\left(C l_{m_{i}}(U)\right) \subseteq \operatorname{Int}_{m_{j}}\left(C l_{m_{i}}\left(m_{i j} s C l(U)\right)\right) \subseteq m_{i j} s C l(U)$.

Definition 2.24. [10] A multifunction is a point-to-set correspondence $F: X \rightarrow Y$ between two topological spaces $\mathrm{X}$ and $\mathrm{Y}$ such that for each point $\mathrm{x}$ of $\mathrm{X}, F(x)$ is a none-void subset of $\mathrm{Y}$.

In the sense of Berge [9], we shall denote and define the upper and lower inverse of a non-void subset $G$ of $Y$ with respect to a multifunction $F$ respectively by:

$$
F^{+}(G)=\{x \in X: F(x) \subseteq G\} \text { and } F^{-}(G)=\{x \in X: F(x) \cap G \neq \varnothing\} .
$$

Generally, $F^{-}$and $F^{+}$between $Y$ and the power set $\mathscr{P}(X)$, $F^{-}(y)=\{x \in X: y \in F(x)\}$ provided $y \in Y$. Clearly for a non-void subset $G$ of $Y, F^{-}(G)=\bigcup\left\{F^{-}(y): y \in G\right\}$ and also,

$$
F^{+}(G)=X \backslash F^{-}(Y \backslash G) \text { and } F^{-}(G)=X \backslash F^{+}(Y \backslash G)
$$

For any non-void subsets $A$ and $G$ of $X$ and $Y$ respectively, $\quad F(A)=\bigcup_{x \in A} F(x)$ and $A \subseteq F^{+}(F(A))$ and also, $F\left(F^{+}(G)\right) \subseteq G$.

Definition 2.25. [2] [12] A multifunction $F:(X, \mathscr{T}) \rightarrow(Y, Q)$, between topological spaces $X$ and $Y$ is said to be:

1) upper irresolute at a point $x_{o}$ of $X$ provided for any semiopen subset $G$ of $Y$ such that $F\left(x_{o}\right) \subseteq G$, there exists a semiopen subset $O$ of $X$ with $x_{o} \in O$ such that $F(O) \subseteq G \quad$ (or $O \subseteq F^{+}(G)$ ).

2) lower irresolute at a point $x_{o}$ of $X$ provided for any semiopen subset $G$ of $Y$ such that $F\left(x_{o}\right) \cap G \neq \varnothing$, there exists a semiopen subset $O$ of $X$ with $x_{o} \in O$ such that $F(x) \cap G \neq \varnothing$ for all $x \in O$ (or $O \subseteq F^{-}(G)$ ).

3 ) upper (resp lower) irresolute provided it is upper (resp lower) irresolute at all points $x_{o}$ of $X$.

Definition 2.26. [11] Let $\left(\left(X, \mathscr{T}_{i}, \mathscr{T}_{j}\right), m_{i j}(X)\right)$ and $\left(\left(Y, \mathscr{T}_{1}, \mathscr{T}_{2}\right), m_{i j}(Y)\right)$, $i, j=1,2 ; i \neq j$ be minimal bitopological spaces. We shall call a multifunction $F:\left(\left(X, \mathscr{T}_{i}, \mathscr{T}_{j}\right), m_{i j}(X)\right) \rightarrow\left(\left(Y, \mathscr{C}_{j}, \mathscr{C}_{i}\right), m_{i j}(Y)\right)$ to be:

1) Upper $m_{i j}$-semi-continuous at some point $x_{o} \in X$ provided for any $m_{i j}(Y)$-open set $V$ satisfying $F\left(x_{o}\right) \subseteq V$, there is an $m_{i j}(X)$-semiopen set $U$ with $x_{o} \in X$ for which $F(U) \subseteq V$ (or $U \subseteq F^{+}(V)$ ).

2) Lower $m_{i j}$-semi-continuous at some point $x_{o} \in X$ provided for each $m_{i j}(Y)$-open set $V$ satisfying $F\left(x_{o}\right) \cap V \neq \varnothing$, we can find an $m_{i j}(X)$-semiopen set $U$ with $x_{o} \in U$ such that for all $z \in U, F(z) \cap V \neq \varnothing$.

3) Upper (resp Lower) $m_{i j}$-semi continuous if it is Upper (resp Lower) $m_{i j}$ -semi continuous at each and every point of $X$. 


\section{Some Properties of Upper and Lower M-Asymmetric Irresolute Multifunctions}

In this section, we introduce and study a special kind of multifunctions for which the inverse of $M$-asymmetric semiopen sets is $M$-asymmetric semiopen.

Definition 3.1. A multifunction

$F:\left(\left(X, \widetilde{\mathscr{T}}_{i}, \mathscr{T}_{j}\right), m_{i j}(X)\right) \rightarrow\left(\left(Y, \mathscr{C}_{i}, \mathscr{Q}_{j}\right), m_{i j}(Y)\right)$ between minimal bitopological spaces $\left(\left(X, \mathscr{T}_{i}, \mathscr{T}_{j}\right), m_{i j}(X)\right)$ and $\left(\left(Y, \mathscr{T}_{1}, \mathscr{T}_{2}\right), m_{i j}(Y)\right), i, j=1,2 ; i \neq j$ shall be called:

1) upper $M$-Asymmetric irresolute at a point $x_{o} \in X$ provided for any $m_{i j}(Y)$-semiopen subset $G$ such that $F\left(x_{o}\right) \subseteq G$, there exists an $m_{i j}(X)$-semiopen set $O$ with $x_{o} \in O$ such that $F(O) \subseteq G$ whence $O \subseteq F^{+}(G)$.

2) lower $M$-Asymmetric irresolute at a point $x_{o} \in X$ provided for any $m_{i j}(Y)$-semiopen set $G$ that intersects $F\left(x_{o}\right)$, there exists a $m_{i j}(X)$-semiopen set $O$ with $x_{o} \in O$ such that $F(x) \cap G \neq \varnothing$ for all $x \in O$ whence $O \subseteq F^{-}(G)$ ).

3) upper (resp lower) $M$-Asymmetric irresolute provided it is upper (resp lower) $M$-Asymmetric irresolute at each and every point $x_{o}$ of $X$.

Clearly, $F^{+}(G)$ and $F^{-}(G)$ are $m_{i j}(X)$-semiopen for any $m_{i j}(Y)$-semiopen set $G$.

Remark 3.2. It should consequently be noted that upper M-asymmetric irresolute and lower M Asymmetric irresolute multifunctions are independent of each other as the example below illustrates.

Example 3.3. Let the minimal structures on $X=\{-2,-1,0,1,2,3\}$ be defined by $m_{1}(X)=\{\varnothing,\{-2\},\{1\},\{-1,0\},\{0,1,3\}, X\}$ and $m_{2}(X)=\{\varnothing,\{1\},\{3\},\{0,1,3\}, X\}$. Also let $m_{1}(Y)=\{\varnothing,\{b\},\{f\},\{g\},\{a, c\},\{e, g\},\{b, d, f, g\}, Y\}$ and $m_{2}(Y)=\{\varnothing,\{b, d\},\{e, g\},\{b, d, f, g\}, Y\}$ be the minimal structure on $Y=\{a, b, c, d, e, f, g\}$. Define a multifunction $F:\left(\left(X, \mathscr{T}_{1}, \mathscr{T}_{2}\right), m_{i j}\right) \rightarrow\left(\left(Y, \mathscr{C}_{1}, \mathscr{C}_{2}\right), m_{i j}\right)$ by:

1)

$$
F(x)= \begin{cases}\{b\}, & x=-2 \\ \{g\}, & x=0 \\ \{a, b\}, & x=1 \\ \{a, e, g\}, & x=3\end{cases}
$$

Then, $F$ is upper $M$-asymmetric irresolute but not lower $M$-asymmetric irresolute, indeed, $\{g\} \in m_{i j} S O(Y)$ but, $F^{-}(\{g\})=\{0,3\} \notin m_{i j} s O(X)$ nor a member of $m_{i j} O(X)$.

2)

$$
F(x)= \begin{cases}\{g\}, & x=-2 \\ \{b\}, & x=0 \\ \{a\}, & x=1 \\ \{a, e, g\}, & x=3\end{cases}
$$


Then, $F$ is lower $M$-asymmetric irresolute but not upper $M$-asymmetric irresolute, since $\{g\} \in m_{i j} S O(Y)$ but, $F^{+}(\{g\})=\{-2\} \notin m_{i j} S O(X)$ nor a member of $m_{i j} O(X)$.

In this part of the section, we discuss some characterizations involving upper $M$-asymmetric irresolute multifunctions.

Theorem 3.4. A multifunction $F:\left(\left(X, \mathscr{T}_{i}, \mathscr{T}_{j}\right), m_{i j}(X)\right) \rightarrow\left(\left(Y, \mathscr{Q}_{i}, \mathscr{Q}_{j}\right), m_{i j}(Y)\right), i, j=1,2 ; i \neq j$ for which $\left(\left(Y, Q_{i}, Q_{j}\right), m_{i j}(Y)\right)$ satisfies property, is said to be upper M-asymmetric irresolute at some point $x_{o}$ of $\mathrm{X}$ if and only if for each $m_{i j}(Y)$-semiopen set $\mathrm{G}$ containing $F\left(x_{o}\right), x_{o} \in C l_{m_{j}}\left(\operatorname{Int}_{m_{i}}\left(F^{+}(G)\right)\right)$.

Proof. For necessity, suppose that $x_{o} \in C l_{m_{j}}\left(\operatorname{Int}_{m_{i}}\left(F^{+}(G)\right)\right)$ for an $m_{i j}(Y)$ -semiopen set $G$ containing $F\left(x_{o}\right)$. Then, by Lemma 2.14 there is some $m_{i j}(X)$-semiopen set $O$ containing the point $x_{o}$ such that $x_{o} \in O \subseteq F^{+}(G)$. Consequently, $F(O) \subseteq G \subseteq C l_{m_{j}}\left(\operatorname{Int}_{m_{i}}(G)\right)$ by the $m_{i j}(Y)$-semiopenness of $G$ and so, $F$ is an upper $M$-asymmetric irresolute multifunction at a point $x_{o}$ in $X$.

For sufficiency, if $F$ is an upper $M$-asymmetric irresolute multifunction at a point $x_{o}$ of $X$ and $G$ is any $m_{i j}(Y)$-semiopen set satisfying $F\left(x_{o}\right) \subseteq G$ then by Definition 3.1, we can find some $m_{i j}(X)$-semiopen set $O$ having $x$ such that $F(O) \subseteq G$. Consequently, $F(O) \subseteq C l_{m_{j}}\left(\operatorname{Int}_{m_{i}}(G)\right)$ since $G$ is $m_{i j}(Y)$-semiopen. Because the semiopenness of $O$ implies $O \subseteq m_{i j} \operatorname{sint}(O)$, the satisfaction of property , Lemma 2.19 (1) and Lemma 2.20 (4) then gives

$$
x_{o} \in O=m_{i j} \operatorname{sint}(O) \subseteq C l_{m_{j}}\left(\operatorname{Int}_{m_{i}}(O)\right) \subseteq C l_{m_{j}}\left(\operatorname{Int}_{m_{i}}\left(F^{+}(G)\right)\right) .
$$

Theorem 3.5. Let $F:\left(\left(X, \mathscr{T}_{i}, \mathscr{T}_{j}\right), m_{i j}(X)\right) \rightarrow\left(\left(Y, \mathscr{C}_{i}, \mathscr{C}_{j}\right), m_{i j}(Y)\right), i, j=1,2$; $i \neq j$ with $\left(\left(Y, Q_{i}, Q_{j}\right), m_{i j}(Y)\right)$ satisfying property $\mathscr{R}$ be a multifunction. $\mathrm{F}$ is upper $\mathrm{M}$-asymmetric irresolute at a point $x_{o}$ of $\mathrm{X}$ if and only if for any $m_{i j}(X)$-semiopen neighbourhood $\mathrm{O}$ of a point $x_{o}$ and any $m_{i j}(Y)$-semiopen set $\mathrm{G}$ containing $F\left(x_{o}\right)$, there exists a none-void $m_{i j}(X)$-open set $O_{G}$ such that $O_{G}$ is a subset of $\mathrm{O}$ and $F\left(O_{G}\right)$ is contained in $\mathrm{G}$.

Proof. For necessity, suppose $F$ is upper $M$-asymmetric irresolute at a point $x_{o}$ of $X$, let $G$ be an $m_{i j}(Y)$-semiopen set for which $F\left(x_{o}\right) \subseteq G$. By Theorem 3.4, $x_{o} \in F^{+}(G) \subseteq C l_{m_{j}}\left(\operatorname{Int}_{m_{i}}\left(F^{+}(G)\right)\right)$. Let $O$ be an $m_{i j}(X)$-semiopen neighbourhood of $x_{o}$, then $F(O) \subseteq G$, whence $O \subseteq F^{+}(G)$ by Definition 3.1 and so, $O \cap \operatorname{Int}_{m_{i}}\left(\operatorname{Int}_{m_{j}}\left(F^{+}(G)\right)\right) \neq \varnothing$. Since $C l_{m_{j}}\left(\operatorname{Int}_{m_{i}}\left(F^{+}(G)\right)\right) \supseteq F^{+}(G) \supseteq \operatorname{Int}_{m_{i}}\left(\operatorname{Int}_{m_{j}}\left(F^{+}(G)\right)\right)$, we obtain from Lemma 2.14 that $O \cap C l_{m_{j}}\left(\operatorname{Int}_{m_{i}}\left(F^{+}(G)\right)\right) \neq \varnothing$. Now, set $O_{G}=O \cap \operatorname{Int}_{m_{i}}\left(\operatorname{Int}_{m_{j}}\left(F^{+}(G)\right)\right)$, then $O_{G}$ is none-void, $O_{G} \subseteq O$, $O_{G} \subseteq \operatorname{Int}_{m_{i}}\left(\operatorname{Int}_{m_{j}}\left(F^{+}(G)\right)\right) \subseteq F^{+}(G)$ whence, $F\left(O_{G}\right) \subseteq G$. Thus, $O_{G}$ is $m_{i j}(X)$-open.

For sufficiency, suppose from $X,\left\{O_{x_{o}}\right\}$ is a family of $m_{i j}(X)$-semiopen neighbourhoods of a point $x_{o}$. Then, for any $m_{i j}(X)$-semiopen set $O$ containing $x_{o}$ and any $m_{i j}(Y)$-semiopen set $G$ containing $F\left(x_{o}\right)$, there is a non- 
empty $m_{i j}(X)$-open set $O_{G}$ contained in $O$ for which $F\left(O_{G}\right) \subseteq G$. Set $Z=\bigcup_{O \in O_{x_{o}}} O_{G}$, then $Z$ is $m_{i j}$-open, $x_{o} \in C l_{m_{i}}\left(C l_{m_{j}}(Z)\right)$ by Theorem 3.4 and $F(Z) \subseteq G$. Set $T=\left\{x_{o}\right\} \cup Z$, then

$$
Z \subseteq T \subseteq C l_{m_{i}}\left(C l_{m_{j}}(Z)\right)
$$

Consequently, $Z$ is an $m_{i j}(X)$-semiopen set, $x_{o} \in T$ and $F(T) \subseteq G$ whence, $T \subseteq F^{+}(G)$ as $T$ is $m_{i j}(X)$-semiopen by Definition 2.6. Thus, $F$ is an upper $M$-asymmetric irresolute multifunction at a point $x_{o}$ of $X$.

Remark 3.6. Theorem 3.5 basically is telling us that, every upper M-asymmetric irresolute multifunction is upper $\mathrm{M}$-asymmetric semicontinuous however, the converse is not necessarily true, as will be shown in an example later.

Theorem 3.7. For a multifunction

$F:\left(\left(X, \mathscr{T}_{i}, \mathscr{T}_{j}\right), m_{i j}(X)\right) \rightarrow\left(\left(Y, Q_{j}, \mathscr{C}_{i}\right), m_{i j}(Y)\right)$ with $\left(\left(Y, Q_{j}, Q_{i}\right), m_{i j}(Y)\right)$, $i, j=1,2 ; i \neq j$ satisfying property $\mathscr{B}$, the properties below are equivalent:

1) $F$ is upper $M$-asymmetric irresolute,

2) For every $m_{i j}(Y)$-semiopen set $G$, the set $F^{+}(G)$ is $m_{i j}(X)$-semiopen;

3) For each $m_{i j}(Y)$-semiclosed set $K$, the set $F^{-}(K)$ is $m_{i j}(X)$-semiclosed;

4) For any set $E$ of $X$, there holds the inclusion

$F\left(m_{i j}(X) s C l(E)\right) \subseteq m_{i j}(Y) s C l(F(E))$;

5) Given any subset $V$ of $Y$, there holds the set inclusion

$F^{-}\left(m_{i j}(Y) s C l(V)\right) \supseteq m_{i j}(X) s C l\left(F^{-}(V)\right)$;

6) Given any subset $Q$ of $Y$, there results

$F^{+}\left(m_{i j}(Y) \operatorname{sint}(Q)\right) \subseteq m_{i j}(X) \operatorname{sint}\left(F^{+}(Q)\right)$.

Proof.

(1) $\Rightarrow$ (2): For if (1) holds, let $x_{o}$ be any point of $X$ and let $G$ be any $m_{i j}(Y)$-semiopen set satisfying $F\left(x_{o}\right) \subseteq G$, so that $x_{o} \in F^{+}(G)$. By the hypothesis, there is some $m_{i j}(X)$-semiopen neighborhood $O$ of $x_{o}$ such that $F(O) \subseteq G$, whence $x \in O \subseteq F^{+}(G)$. By Theorem 3.4, $x_{o} \in C l_{m_{i}}\left(\operatorname{Int}_{m_{j}}\left(F^{+}(G)\right)\right)$ and so,

$$
F^{+}(G) \subseteq C l_{m_{j}}\left(\operatorname{Int}_{m_{i}}\left(F^{+}(G)\right)\right)=m_{i j}(X) \operatorname{sint}\left(F^{+}(G)\right) .
$$

By Definition 2.6 and then Lemma 2.13, $F^{+}(G)$ is $m_{i j}(X)$-semiopen.

(2) $\Rightarrow$ (3): Suppose (2) holds. Let $K$ be an $m_{i j}(Y)$-semiclosed set. Because $Y \backslash K$ is $m_{i j}(Y)$-semiopen, $F^{+}(Y \backslash K)=X \backslash F^{-}(K)$ and $F^{-}(Y \backslash K)=X \backslash F^{+}(K)$, we have from Lemma 2.13, Lemma 2.15 and Lemma 2.19 that,

$$
\begin{aligned}
X \backslash m_{i j}(X) s C l\left(F^{-}(K)\right) & =m_{i j}(X) \operatorname{sint}\left(X \backslash F^{-}(K)\right) \\
& =m_{i j}(X) \operatorname{sint}\left(F^{+}(Y \backslash K)\right) \\
& =F^{+}(Y \backslash K) \\
& =X \backslash F^{-}(K) .
\end{aligned}
$$


And the result holds true provided $m_{i j}(X) s C l\left(F^{-}(K)\right)=F^{-}(K)$, so that $F^{-}(K)$ is $m_{i j}(X)$-semiclosed.

(3) $\Rightarrow$ (4): If (3) holds, let $E$ be some $m_{i j}(X)$-semiopen set. Then by Definition 2.11,

$$
\begin{aligned}
m_{i j}(X) s C l(E) & =\bigcap\left\{J: E \subseteq J \text { and } J \in m_{i j} s C(X)\right\} \\
& =\bigcap\left\{F^{-}(K): E \subseteq F^{-}(K) \text { and } F^{-}(K) \in m_{i j} s C(X)\right\} \\
& \subseteq F^{-}\left(\bigcap\left\{K: F(E) \subseteq K \text { and } K \in m_{i j} s C(Y)\right\}\right) \\
& =F^{-}\left(m_{i j}(Y) s C l(F(E))\right) .
\end{aligned}
$$

Thus, $F\left(m_{i j}(X) s C l(E)\right) \subseteq m_{i j}(Y) s C l(F(E))$.

(4) $\Rightarrow$ (5): Since for any subset $V$ of $Y, m_{i j}(Y) s C l(V)$ is an $m_{i j}(Y)$-semiclosed set, we obtain from the closure property that,

$$
\begin{aligned}
& m_{i j}(X) s C l\left(F^{-}(V)\right)=\bigcap\left\{P: F^{-}(V) \subseteq P \text { and } P \in m_{i j} s C(X)\right\} \\
& =\bigcap\left\{F^{-}(K): F^{-}(V) \subseteq F^{-}(K) \text { and } F^{-}(K) \in m_{i j} s C(X)\right\} \\
& \subseteq F^{-}\left(\bigcap\left\{K: V \subseteq K \text { and } K \in m_{i j} s C(Y)\right\}\right) \\
& =F^{-}\left(m_{i j}(Y) s C l(V)\right) .
\end{aligned}
$$

As a result, $m_{i j}(X) s C l\left(F^{-}(V)\right) \subseteq F^{-}\left(m_{i j}(Y) s C l(V)\right)$.

(5) $\Rightarrow$ (6): For any subset $Q$ of $Y, m_{i j}(Y) \operatorname{sint}(Q)=Y \backslash m_{i j}(Y) s C l(Y \backslash Q)$. For if (5) holds, Lemma 2.13 implies

$$
\begin{aligned}
X \backslash m_{i j}(X) \operatorname{sint}\left(F^{+}(Q)\right) & =m_{i j}(X) s C l\left(X \backslash F^{+}(Q)\right) \\
& =m_{i j}(X) s C l\left(F^{-}(Y \backslash Q)\right) \\
& \subseteq F^{-}\left(m_{i j}(Y) s C l(Y \backslash Q)\right) \\
& =F^{-}\left(Y \backslash m_{i j}(Y) \operatorname{sint}(Q)\right) \\
& =X \backslash F^{+}\left(m_{i j}(Y) \operatorname{sint}(Q)\right) .
\end{aligned}
$$

Consequently, $F^{+}\left(m_{i j}(Y) \operatorname{sint}(Q)\right) \subseteq m_{i j}(X) \operatorname{sint}\left(F^{+}(Q)\right)$.

(6) $\Rightarrow$ (1): For if (6) holds, let $x_{o}$ be any point of $X$ and $G$ be any $m_{i j}(Y)$ -semopen neighborhood of $F^{+}\left(x_{o}\right)$. From (2), $F^{+}(G) \subseteq C l_{m_{j}}\left(\operatorname{Int}_{m_{i}}\left(F^{+}(G)\right)\right)$ is an $m_{i j}(X)$-semiopen set containing $x_{o}$. Letting $O=F^{+}(G)$ gives $F(O) \subseteq G$, as a result, $F$ is upper $m_{i j}$-asymmetric irresolute at $x_{o}$.

Theorem 3.8. Let $F:\left(\left(X, \mathscr{T}_{i}, \mathscr{T}_{j}\right), m_{i j}(X)\right) \rightarrow\left(\left(Y, \mathscr{C}_{i}, \mathscr{C}_{j}\right), m_{i j}(Y)\right), i, j=1,2$; $i \neq j$ with $\left(\left(Y, \mathscr{C}_{i}, Q_{j}\right), m_{i j}(Y)\right)$ satisfying property $\mathscr{B}$ be an upper M-asymmetric irresolute multifunction at an arbitrary point $x_{o}$ of X. Then, the properties below hold:

1) $F^{+}(Q)$ is an $m_{i j}(X)$-semi neighbourhood of $x_{o}$ for any arbitrary $m_{i j}(Y)$-semi neighbourhood $Q$ of $F\left(x_{o}\right)$.

2) There exists an $m_{i j}(X)$-semi neighbourhood $R$ of $x_{o}$ such that $F(R) \subseteq Q$ for each $m_{i j}(Y)$-semi neighbourhood $Q$ of $F\left(x_{o}\right)$.

\section{Proof.}

1) Let $x_{o}$ be any point of $X$ and $Q$ be an $m_{i j}(Y)$-semi neighbourhood of 
$F\left(x_{o}\right)$. By Definition 2.10, there is an $m_{i j}(Y)$-semiopen set $V$ such that $V \subseteq Q$ and so, $F\left(x_{o}\right) \subseteq V \subseteq Q$. Since $F$ is upper irresolute,

$x_{o} \subseteq F^{+}(V) \subseteq F^{+}(Q)$. Because, $F^{+}(V) \in m_{i j} s O(X), F^{+}(Q)$ is an $m_{i j}(Y)$ -semi neighbourhood of $x_{0}$.

2) Clearly follows from (1). Indeed, for any point $x_{o}$ of $X$ and $m_{i j}(Y)$-semi neighbourhood $Q$ of $F\left(x_{o}\right)$ put $R=F^{+}(Q)$. From (1), $R$ is $m_{i j}(X)$-semi neighbourhood of $x_{o}$ and by the hypothesis, $F(R) \subseteq Q$.

In this part of the section, we discuss some characterizations involving lower $M$-asymmetric irresolute multifunctions.

Theorem 3.9. A multifunction

$F:\left(\left(X, \mathscr{T}_{i}, \mathscr{T}_{j}\right), m_{i j}(X)\right) \rightarrow\left(\left(Y, \mathscr{C}_{i}, \mathscr{C}_{j}\right), m_{i j}(Y)\right), i, j=1,2 ; i \neq j$ for which $\left(\left(Y, C_{i}, C_{j}\right), m_{i j}(Y)\right)$ satisfies property $\mathscr{B}$, is said to be lower M-asymmetric irresolute at a point $x_{o}$ of $\mathrm{X}$ if and only if for every $m_{i j}(Y)$-semiopen set $\mathrm{G}$ intersecting $F\left(x_{o}\right), x_{o} \in C l_{m_{j}}\left(\operatorname{Int}_{m_{i}}\left(F^{-}(G)\right)\right)$.

Proof. For necessity, suppose $F$ is a lower $M$-asymmetric irresolute multifunction at some point $x_{o}$ of $X$. Let $G$ be an $m_{i j}(Y)$-semiopen set intersecting $F\left(x_{o}\right)$. By hypothesis, there is an $m_{i j}(X)$-semiopen set $O$ having $x_{o}$ such that $G$ intersects $F(x)$ for each $x$ in $O$ whence, $x \in O \subseteq F^{-}(G)$. As $O$ is $m_{i j}(X)$ -semiopen, $O=m_{i j} \operatorname{sint}(O) \subseteq C l_{m_{j}}\left(\operatorname{Int}_{m_{i}}(O)\right)$. Thus, from Lemma 2.19 and Lemma 2.20, we obtain

$$
x \in O=m_{i j}(X) \operatorname{sint}(O) \subseteq C l_{m_{j}}\left(\operatorname{Int}_{m_{i}}(O)\right) \subseteq C l_{m_{j}}\left(\operatorname{Int}_{m_{i}}\left(F^{-}(G)\right)\right) .
$$

For sufficiency, let $G$ be an $m_{i j}(Y)$-semiopen set intersecting with $F\left(x_{o}\right)$. From Lemma 2.14 and the assumption, $x_{o} \in C l_{m_{j}}\left(\operatorname{Int}_{m_{i}}\left(F^{-}(G)\right)\right)$. By Definition 3.1 (2), there is an $m_{i j}(X)$-semiopen set $O$ of $X$ containing $x_{o}$ such that, $G$ intersects $F(x)$ for each $x$ belonging to $O$. Thus, $O \subseteq F^{-}(G)$, whence $F(O) \subseteq G$. Since $G$ is $m_{i j}(Y)$-semiopen, so is $F^{-}(G)$ and hence, $F$ is a lower $M$-asymmetric irresolute multifunction at a point $x_{o}$ of $X$.

Theorem 3.10. Let $F:\left(\left(X, \mathscr{T}_{i}, \mathscr{T}_{j}\right), m_{i j}(X)\right) \rightarrow\left(\left(Y, \mathscr{C}_{i}, Q_{j}\right), m_{i j}(Y)\right)$, $i, j=1,2 ; i \neq j$ with $\left(\left(Y, \mathscr{C}_{i}, \mathscr{C}_{j}\right), m_{i j}(Y)\right)$ satisfying property $\mathscr{B}$ be a multifunction. $\mathrm{F}$ is lower $\mathrm{M}$-asymmetric irresolute at a point $x_{o}$ of $\mathrm{X}$ if and only if for any $m_{i j}(X)$-semiopen neighbourhood $\mathrm{O}$ of a point $x_{o}$ and any $m_{i j}(Y)$ -semiopen set $\mathrm{G}$ intersecting $F\left(x_{o}\right)$, there exists a nonempty $m_{i j}(X)$-open set $O_{G}$ such that $O_{G}$ is contained in $\mathrm{O}$ and $F(x)$ intersects $\mathrm{G}$ for every point $\mathrm{x}$ in $O_{G}$.

Proof. For necessity, let $F$ be lower $M$-asymmetric irresolute at a point $x_{o}$ of $X$ and $G$ be an $m_{i j}(Y)$-semiopen set satisfying $F\left(x_{o}\right) \cap G$. By Theorem 3.10, $x_{o} \in F^{-}(G) \subseteq C l_{m_{j}}\left(\operatorname{Int}_{m_{i}}\left(F^{-}(G)\right)\right)$. Let $O$ be any $m_{i j}(X)$-semiopen neighbourhood of $x_{o}$. Since $F\left(x_{o}\right) \cap G \neq \varnothing, O \subseteq F^{-}(G)$ and so,

$O \cap \operatorname{Int}_{m_{i}}\left(\operatorname{Int}_{m_{j}}\left(F^{-}(G)\right)\right) \neq \varnothing$. Because

$\operatorname{Int}_{m_{i}}\left(\operatorname{Int}_{m_{j}}\left(F^{-}(G)\right)\right) \subseteq F^{-}(G) \subseteq C l_{m_{j}}\left(\operatorname{Int}_{m_{i}}\left(F^{-}(G)\right)\right)$,

$O \cap C l_{m_{j}}\left(\operatorname{Int}_{m_{i}}\left(F^{+}(G)\right)\right) \neq \varnothing$. Define $O_{G}$ by $O_{G}=O \cap \operatorname{Int}_{m_{i}}\left(\operatorname{Int}_{m_{j}}\left(F^{-}(G)\right)\right)$, then $O_{G}$ is nonempty, $O_{G} \subseteq O$ and $F(x) \cap G \neq \varnothing$ for all points $x \in O_{G}$. 
Consequently, $O_{G}$ is $m_{i j}(X)$-open.

For sufficiency, suppose $\left\{O_{x_{o}}\right\}$ is a family of $m_{i j}(X)$-semiopen neighbourhoods of a point $x_{o}$. Then, for any $m_{i j}$-semiopen set $O$ of $X$ containing $x_{o}$ and any $m_{i j}(Y)$-semiopen set $G$ intersecting $F\left(x_{o}\right)$, there is a nonempty $m_{i j}(X)$-open set $O_{G}$ contained in $O$ for which $F(x)$ intersects $G$ for all $x$ contained in $O_{G}$. Let $Z=\bigcup_{O \in O_{x_{o}}} O_{G}$, then $Z$ is $m_{i j}(X)$-open, $x_{o}$ is contained in $C l_{m_{i}}\left(C l_{m_{j}}(Z)\right)$ by Theorem 3.10 and $F(x)$ intersects $G$ for all $x \in Z$. Set $T=\left\{x_{o}\right\} \cup Z$, then

$$
Z \subseteq T \subseteq C l_{m_{i}}\left(C l_{m_{j}}(Z)\right)
$$

Consequently, $Z$ is an $m_{i j}(X)$-semiopen set, $x_{o} \in T$ and $F(x)$ intersects $G$ for all $x$ in $T$, whence $T \subseteq F^{-}(G)$. Thus, $F$ is a lower $M$-asymmetric irresolute multifunction at a point $x_{o}$ in $X$.

Theorem 3.11. If $\left(\left(Y, C_{i}, Q_{j}\right), m_{i j}(Y)\right), i, j=1,2 ; i \neq j$, satisfies property $\mathscr{B}$, then the following properties are equivalent for a multifunction $F:\left(\left(X, \mathscr{T}_{i}, \mathscr{T}_{j}\right), m_{i j}(X)\right) \rightarrow\left(\left(Y, \mathscr{C}_{i}, \mathscr{C}_{j}\right), m_{i j}(Y)\right):$

1) $F$ is lower $M$-asymmetric irresolute;

2) For every $m_{i j}(Y)$-semiopen set $G$, the set $F^{-}(G)$ is $m_{i j}(X)$-semiopen;

3) For any $m_{i j}(Y)$-semiclosed set $K$, the set $F^{+}(K)$ is $m_{i j}(X)$-semiclosed;

4) Given any subset $V$ of $Y, \quad F^{+}\left(m_{i j}(Y) s C l(V)\right) \supseteq m_{i j}(X) s C l\left(F^{+}(V)\right)$;

5) Given any subset $U$ of $X$, there holds the set inclusion $F\left(m_{i j}(X) s C l(U)\right) \subseteq m_{i j}(Y) s C l(F(U))$;

6) Given any subset $W$ of $Y$, the inclusion $F^{-}\left(m_{i j}(Y) \operatorname{sint}(W)\right) \subseteq m_{i j}(X) \operatorname{sint}\left(F^{-}(W)\right)$ holds true.

\section{Proof.}

(1) $\Rightarrow$ (2): For if $F$ is lower $m_{i j}$-asymmetric irresolute at a point $x_{o}$ of $X$, let $G$ be any $m_{i j}(Y)$-semiopen set satisfying $F\left(x_{o}\right) \cap G \neq \varnothing$ and so $x_{o} \in F^{-}(G)$. By Theorem 3.10, $x \in C l_{m_{i}}\left(\operatorname{Int}_{m_{j}}\left(F^{-}(G)\right)\right)$. The semi-openness of $G$ and arbitrary selection of $x$ in $F^{-}(G)$ then implies $F^{-}(G) \subseteq C l_{m_{i}}\left(\operatorname{Int}_{m_{j}}\left(F^{-}(G)\right)\right)$. Consequently, Definition $2.6(1)$ implies $F^{-}(G)$ is $m_{i j}(X)$-semiopen.

(2) $\Rightarrow$ (3): For if $K$ is any $m_{i j}(Y)$-semiclosed set, we then have that, $Y-K \in m_{i j} s O(Y), \quad F^{-}(Y-K)=X-F^{+}(K)$ and $F^{+}(Y-K)=X-F^{-}(K)$. From Lemma 2.13, Lemma 2.15 and Lemma 2.19, we obtain

$$
\begin{aligned}
X \backslash m_{i j}(X) s C l\left(F^{+}(K)\right) & =m_{i j}(X) \operatorname{sint}\left(X \backslash F^{+}(K)\right) \\
& =m_{i j}(X) \operatorname{sint}\left(F^{-}(Y \backslash K)\right) \\
& =F^{-}(Y \backslash K) \\
& =X \backslash F^{+}(K) .
\end{aligned}
$$

Consequently, $m_{i j}(X) s C l\left(F^{+}(K)\right)=F^{+}(K)$ and so, $F^{+}(K)$ is $m_{i j}(X)$-semiclosed.

(3) $\Rightarrow$ (4): Let $V$ be any subset of $Y$, then $m_{i j}(Y) s C l(V)$ is $m_{i j}(Y)$-semiclosed by Lemma 2.13 and Lemma 2.20. For if (4) holds, then 
$F^{+}\left(m_{i j}(Y) s C l(V)\right)$ is $m_{i j}(X)$-semiclosed and so,

$$
\begin{aligned}
& F^{+}\left(m_{i j}(Y) s C l(V)\right)=F^{+}\left(\bigcap\left\{K: V \subseteq K \text { and } K \in m_{i j} s C(Y)\right\}\right) \\
& \supseteq \bigcap\left\{F^{+}(K): F^{+}(V) \subseteq F^{+}(K) \text { and } F^{+}(K) \in m_{i j} s C(X)\right\} \\
& =m_{i j}(X) s C l\left(F^{+}(V)\right) .
\end{aligned}
$$

There results $F^{+}\left(m_{i j}(Y) s C l(V)\right) \supseteq m_{i j}(X) s C l\left(F^{+}(V)\right)$.

(4) $\Rightarrow$ (5): Suppose (4) holds, let $U$ be any subset of $X$. Put $V=F(U)$, then $U \subseteq F^{+}(V)$. Since $U \subseteq F^{+}(F(U))$, it follows that

$U \subseteq F^{+}\left(m_{i j}(Y) s C l(F(U))\right)$. But then, $m_{i j}(Y) s C l(F(U)) \in m_{i j} s C(Y)$ and so, by the hypothesis, $F^{+}\left(m_{i j}(Y) s C l(F(U))\right) \in m_{i j} s C(X)$. Clearly,

$m_{i j}(X) s C l(U) \subseteq F^{+}\left(m_{i j}(Y) s C l(F(U))\right)$; indeed,

$$
\begin{aligned}
& m_{i j}(X) s C l(U) \subseteq m_{i j}(X) s C l\left(F^{+}(V)\right) \\
& =\bigcap\left\{F^{+}(K): F^{+}(V) \subseteq F^{+}(K) \text { and } F^{+}(K) \in m_{i j} s C(X)\right\} \\
& \subseteq F^{+}\left(\bigcap\left\{K: V \subseteq K \text { and } K \in m_{i j} s C(Y)\right\}\right) \\
& =F^{+}\left(\bigcap\left\{K: F(U) \subseteq K \text { and } K \in m_{i j} s C(Y)\right\}\right) \\
& =F^{+}\left(m_{i j}(Y) s C l(F(U))\right) .
\end{aligned}
$$

Consequently,

$F\left(m_{i j}(X) s C l(U)\right) \subseteq F\left(F^{+}\left(m_{i j}(Y) s C l(F(U))\right)\right)=m_{i j}(Y) s C l(F(U))$.

$(5) \Rightarrow$ (6): Suppose (5) holds, let $W$ be any arbitrary subset of $Y$. Since

$m_{i j}(Y) \operatorname{sint}(W)=Y \backslash m_{i j}(Y) s C l(Y \backslash W), F^{-}(Y-W)=X-F^{+}(W)$ and $F^{+}(Y-W)=X-F^{-}(W)$, we get:

$$
\begin{aligned}
m_{i j}(X) \operatorname{sint}\left(F^{-}(W)\right) & =X \backslash m_{i j}(X) s C l\left(X \backslash F^{-}(W)\right) \\
& =X \backslash m_{i j}(X) s C l\left(F^{+}(Y \backslash W)\right) \\
& \supseteq X \backslash F^{+}\left(m_{i j}(Y) s C l(Y \backslash W)\right) \\
& =F^{-}\left(Y \backslash m_{i j}(Y) s C l(Y \backslash W)\right) \\
& =F^{-}\left(m_{i j}(Y) \operatorname{sint}(W)\right) .
\end{aligned}
$$

Thus, $m_{i j}(X) \operatorname{sint}\left(F^{-}(W)\right) \supseteq F^{-}\left(m_{i j}(Y) \operatorname{sint}(W)\right)$.

(6) $\Rightarrow$ (1): For if (6) holds true, let $x_{o}$ be an arbitrary point in $X$ and let $G$ be any $m_{i j}(Y)$-semiopen set satisfying $F(x) \cap G \neq \varnothing$ whence, $x \in F^{-}(G)$. Since $Y$ satisfies property $\mathscr{R}$, Lemma $2.20(1)$ and Lemma 2.21 implies $G=m_{i j}(Y) \operatorname{sint}(G)$ and so,

$$
F^{-}(G)=F^{-}\left(m_{i j}(Y) \operatorname{sint}(G)\right) \subseteq m_{i j}(X) \operatorname{sint}\left(F^{-}(G)\right) .
$$

Because, $G$ is arbitrary and $x_{o}$ is an arbitrary point in $F^{-}(G)$, we can find an $m_{i j}(X)$-semiopen neighborhood $O$ of $x_{o}$ such that $F(x) \cap G \neq \varnothing$ whence, $x \in F^{-}(G)$ for all $x$ contained in $O$. Consequently, $F^{-}(G)$ is an $m_{i j}(X)$ -semiopen set and so, $F$ is a lower $m_{i j}$-asymmetric irresolute at a point $x_{o}$ and hence, at all points in $X$.

Theorem 3.12. Let $F:\left(\left(X, \mathscr{T}_{i}, \mathscr{T}_{j}\right), m_{i j}(X)\right) \rightarrow\left(\left(Y, \mathscr{C}_{i}, \mathscr{C}_{j}\right), m_{i j}(Y)\right), i, j=1,2$; 
$i \neq j$ with $\left(\left(Y, \mathscr{C}_{i}, \mathscr{Q}_{j}\right), m_{i j}(Y)\right)$ satisfying property $\mathscr{R}$ be a lower M-asymmetric irresolute multifunction at a point $x_{o}$ of $\mathrm{X} . F^{-}(G)$ is $m_{i j}(X)$-semiopen if and only if for every $m_{i j}(Y)$-semiopen set $\mathrm{G}$, there exists an $m_{i j}(X)$-semiopen set $\mathrm{O}$ for which $x_{o}$ lies in $\mathrm{O}$ and $f(x)$ intersects $\mathrm{G}$ for all $\mathrm{x}$ in $\mathrm{O}$.

Proof. For necessity, let $G$ be any $m_{i j}(Y)$-semiopen set and $x_{o}$ be any point in $X$ such that $F\left(x_{o}\right) \cap G \neq \varnothing$, whence $x_{o} \in F^{-}(G) \in m_{i j} s O(X)$. Put $O=F^{-}(G)$, consequently $x$ belongs to $O$ and so by hypothesis, $F(x) \cap G \neq \varnothing$ for all $x$ belonging to $O$, whence $F(O) \subseteq G$.

For sufficiency, suppose $G$ is any $m_{i j}(Y)$-semiopen set and $x_{o}$ is any point in $X$ for which $f\left(x_{o}\right) \cap G$, and so $x_{o} \in F^{-}(G)$. By the hypothesis, there exists an $m_{i j}(X)$-semiopen neighborhood $O$ of $x_{o}$ such that, $F(x) \cap G \neq \varnothing$ for every $x$ in $O$. Set $F^{-}(G)=\bigcup_{x \in F^{-}(G)} O$, then by Lemma 2.8, $F^{-}(G)$ is contained
in $m_{i j} S O(X)$.

The theorem below follows as a consequence of Lemma 2.23 and Theorem 3.12:

Theorem 3.13. Let $F:\left(\left(X, \mathscr{T}_{i}, \mathscr{T}_{j}\right), m_{i j}(X)\right) \rightarrow\left(\left(Y, \swarrow_{i}, Q_{j}\right), m_{i j}(Y)\right), i, j=1,2$; $i \neq j$ with $\left(\left(Y, \mathscr{C}_{i}, \mathscr{C}_{j}\right), m_{i j}(Y)\right)$ satisfying property $\mathscr{B}$ be a multifunction. Then, the statements that follow are equivalent:

1) $F$ is lower $M$-asymmetric irresolute;

2) For any $G$ an $m_{i j}(Y)$-semiopen set, $F^{-}(G) \subseteq C l_{m_{j}}\left(\operatorname{Int}_{m_{i}}\left(F^{-}(G)\right)\right)$;

3) For any $K$ an $m_{i j}(Y)$-semiclosed set, $\operatorname{Int}_{m_{j}}\left(C l_{m_{i}}\left(F^{+}(K)\right)\right) \subseteq F^{+}(K)$;

4) For $U$ a subset of $X, F\left(\operatorname{Int}_{m_{j}}\left(C l_{m_{i}}(U)\right)\right) \subseteq m_{i j}(Y) s C l(F(U))$;

5) For any subset $V$ of $Y, \operatorname{Int}_{m_{j}}\left(C_{m_{i}}\left(F^{+}(V)\right)\right) \subseteq F^{+}\left(m_{i j}(Y) s C l(V)\right)$;

6) For any subset $W$ of $Y, F^{-}\left(m_{i j}(Y) \operatorname{sint}(W)\right) \subseteq C l_{m_{j}}\left(\operatorname{Int}_{m_{j}}\left(F^{-}(W)\right)\right)$.

\section{Proof.}

(1) $\Rightarrow$ (2): For any point $x_{o}$ of $X$, let $G$ be any $m_{i j}(Y)$-semiopen set. From Theorem 3.12 and Lemma 2.23, we obtain

$$
\begin{aligned}
x_{o} & \in m_{i j}(X) \operatorname{sInt}(O) \subseteq m_{i j}(X) \operatorname{sint}\left(F^{-}(G)\right) \\
& \subseteq C l_{m_{j}}\left(\operatorname{Int}_{m_{i}}\left(m_{i j} \operatorname{sint}\left(F^{-}(G)\right)\right)\right) \\
& \subseteq C l_{m_{j}}\left(\operatorname{Int}_{m_{i}}\left(F^{-}(G)\right)\right)
\end{aligned}
$$

Thus, $F^{-}(G) \subseteq C l_{m_{j}}\left(\operatorname{Int}_{m_{i}}\left(F^{-}(G)\right)\right)$.

(2) $\Rightarrow$ (3): For if $K$ is an $m_{i j}(Y)$-semiclosed set, then $Y \backslash K$ is an $m_{i j}(Y)$ -semiopen set. Theorem 3.12 then implies

$$
\begin{aligned}
X \backslash m_{i j}(X) \operatorname{sCl}\left(F^{+}(K)\right) & =m_{i j}(X) \operatorname{sint}\left(X \backslash F^{+}(K)\right) \\
& =m_{i j}(X) \operatorname{sint}\left(F^{-}(Y \backslash K)\right) \\
& \subseteq F^{-}(Y \backslash K) \\
& =X \backslash F^{+}(K) .
\end{aligned}
$$

Since $m_{i j}(X) s C l\left(F^{+}(K)\right)=\operatorname{Int}_{m_{j}}\left(C l_{m_{i}}\left(F^{+}(K)\right)\right)$, we obtain from Lemma 2.23 that, $\operatorname{Int}_{m_{j}}\left(C l_{m_{i}}\left(F^{+}(K)\right)\right) \subseteq F^{+}(K)$.

(3) $\Rightarrow$ (4): If (3) holds, then for any none-void subset $U$ of $X$, we obtain 
from Theorem 3.12 and Lemma 2.23 that,

$$
\operatorname{Int}_{m_{j}}\left(C l_{m_{i}}(U)\right) \subseteq \operatorname{Int}_{m_{j}}\left(C l_{m_{i}}\left(m_{i j}(X) s C l(U)\right)\right) \subseteq F^{-}\left(m_{i j}(Y) s C l(F(U))\right)
$$

Consequently, $F\left(\operatorname{Int}_{m_{j}}\left(C l_{m_{i}}(U)\right)\right) \subseteq C l_{m_{i}}(F(A))$.

$(4) \Rightarrow$ (5): If (4) holds, we have for any subset $V$ of $Y$ and the closure property that,

$$
\begin{aligned}
& m_{i j}(X) s C l\left(F^{+}(V)\right)=\bigcap\left\{E: F^{+}(V) \subseteq E \text { and } E \in m_{i j} s C(X)\right\} \\
& \subseteq \bigcap\left\{F^{+}(K): F^{+}(V) \subseteq F^{+}(K) \text { and } F^{+}(K) \in m_{i j} s C(X)\right\} \\
& =F^{+}\left(\bigcap\left\{K: V \subseteq K \text { and } K \in m_{i j} s C(Y)\right\}\right) \\
& =F^{+}\left(m_{i j}(Y) s C l(V)\right) .
\end{aligned}
$$

Thus by Lemma 2.23, $\operatorname{Int}_{m_{j}}\left(C l_{m_{i j}}\left(F^{+}(V)\right)\right) \subseteq F^{+}\left(m_{i j}(Y) s C l(V)\right)$.

(5) $\Rightarrow$ (6): For if (5) holds, Theorem 3.12 and Lemma 2.13 gives

$$
\begin{aligned}
F^{-}\left(m_{i j}(Y) \operatorname{sint}(W)\right) & =F^{-}\left(Y \backslash m_{i j}(Y) s C l(Y \backslash W)\right) \\
& =X \backslash F^{+}\left(m_{i j}(Y) s C l(Y \backslash W)\right) \\
& \subseteq X \backslash m_{i j}(X) s C l\left(F^{+}(Y \backslash W)\right) \\
& =X \backslash m_{i j}(X) s C l\left(X \backslash F^{-}(B)\right) \\
& =m_{i j}(X) \operatorname{sint}\left(F^{-}(W)\right) .
\end{aligned}
$$

Consequently, Lemma 2.23 implies

$F^{-}\left(m_{i j}(Y) \operatorname{sint}(W)\right) \subseteq C l_{m_{j}}\left(\operatorname{Int}_{m_{j}}\left(F^{-}(W)\right)\right)$.

$(6) \Rightarrow$ (4): Suppose (6) holds. Let $x_{o}$ be any point of $X$ and $G$ be any $m_{i j}(Y)$-semiopen set satisfying $F\left(x_{o}\right) \cap G \neq \varnothing$, so that $x_{o} \in F^{-}(G)$. By Theorem 3.10, $x \in C l_{m_{j}}\left(\operatorname{Int}_{m_{i}}\left(F^{-}(W)\right)\right)$, and so,

$$
F^{-}(G) \subseteq C l_{m_{j}}\left(\operatorname{Int}_{m_{i}}\left(F^{-}(G)\right)\right) .
$$

Thus, Theorem 3.13 implies $F^{-}(G)$ is an $m_{i j}(X)$-semiopen, hence, $F$ is lower $M$-asymmetric irresolute at $x_{o}$.

Remark 3.14. In the examples that follow, it shall clearly be understood that, upper (lower) $\mathrm{M}$-asymmetric irresolute multifunctions are upper (respe lower) M-asymmetric semicontinuous, but the converse does not necessarily hold.

Example 3.15. Let the minimal structures on $X=\{-2,-1,0,1,2,3\}$ be defined by $m_{1}(X)=\{\varnothing,\{0\},\{-2\},\{3\},\{0,1,3\}, X\}$ and $m_{2}(X)=\{\varnothing,\{-1\},\{1\},\{2\},\{0,1,3\}, X\}$ and also on $Y=\{a, b, c, d, e, f, g\}$ be defined by by $m_{1}(Y)=\{\varnothing,\{b\},\{f\},\{g\},\{a, c\},\{e, g\},\{b, d, f, g\}, X\}$ and $m_{2}(Y)=\{\varnothing,\{e\},\{b, d\},\{e, g\},\{b, d, f, g\}, X\}$. Define a multifunction $F:\left(\left(X, \mathscr{T}_{1}, \mathscr{T}_{2}\right), m_{i j}\right) \rightarrow\left(\left(Y, \mathscr{C}_{1}, \mathscr{C}_{2}\right), m_{i j}\right)$ by:

$$
F(x)= \begin{cases}\{g\}, & x=-2 \\ \{a, b\}, & x=0 \\ \{e, g\}, & x=3\end{cases}
$$

1) Then, $F$ is upper $M$-asymmetric irresolute, since 
$F(-2) \subseteq\{c, f, g\} \in m_{i j} s O(Y), \quad F(0) \subseteq\{a, b, c, d\} \in m_{i j} s O(Y)$ and

$F(3) \subseteq\{a, e, g\} \in m_{i j} s O(Y)$ or $F^{+}(\{g\})=\{-2\}$ and $F^{+}(\{a, b\})=\{0\}$ which both belong to $m_{i j} S O(X)$. Fence, $F$ is alos upper $M$-asymmetric semicontinuous.

2) Also observe that, $F(-2) \cap\{a, e, g\} \neq \varnothing, \quad F(0) \cap\{a, e, g\} \neq \varnothing$ and $F(3) \cap\{a, e, g\} \neq \varnothing \quad$ for all $x \in\{-2,0,3\} \in m_{i j} s O(X)$ or $F^{-}(\{g\})=\{-2,3\}$ and $F^{-}(\{a, b\})=\{0\}$ which belong to $m_{i j} s O(X)$. Consequently, $F$ is lower $M$-asymmetric irresolute and hence, lower $M$-asymmetric semicontinuous.

Example 3.16. Let the minimal structures on $X=\{-1,0,1\}$ be defined by $m_{1}(X)=\{\varnothing,\{0\},\{-1\},\{-1,0\}, X\}$ and $m_{2}(X)=\{\varnothing,\{0\},\{-1\},\{-1,0\}, X\}$ and also on $Y=\{a, b, c, d, e\}$ be defined by $m_{1}(Y)=\{\varnothing,\{a\},\{b\},\{b, c, d, e\}, Y\}$ and $m_{2}(Y)=\{\varnothing,\{b\},\{b, c\},\{b, c, d, e\}, Y\}$. Define a multifunction $F:\left(\left(X, \widetilde{T}_{1}, \mathscr{T}_{2}\right), m_{i j}\right) \rightarrow\left(\left(Y, \mathscr{C}_{1}, \mathscr{C}_{2}\right), m_{i j}\right)$ by:

$$
F(x)= \begin{cases}\{b\}, & x=-1 \\ \{b, c\}, & x=0 \\ \{a, d, e\}, & x=1\end{cases}
$$

Then, $F$ is upper and lower $M$-asymmetric semicontinuous, but neither upper nor lower $M$-asymmetric irresolute respectively. Indeed; $\{b\} \in m_{i j} O(Y)$ but $F^{+}(\{b\})=\{-1\} \notin m_{i j} s O(X)$ and also, $\{a, c\} \in m_{i j} s O(Y)$ but, $F^{-}(\{b, c\})=\{-1,0\} \notin m_{i j} s O(X)$.

\section{Conclusion}

In this paper, we have successfully introduced and investigate some properties of a new class of irresolute multifunctions, the upper (lower) $M$-asymmetric irresolute multifunction defined between bitopological spaces with sets satisfying minimal structures. Our work, is a generalization of ideas by Crossley and Hildebrand [1] and, Popa [2], during which we have observed that upper (lower) $M$-asymmetric irresolute multifunctions have their properties similar to those of upper (lower) irresolute multifunctions defined between topological spaces, with the only difference that, in this scenario, asymmetric sets have been used. Secondly, we have noted that the upper (lower) $M$-asymmetric irresolute multifunctions are respectively upper (lower) $M$-asymmetric semicontinuous, however, the converse is not necessarily true as shown in the counter Examples 3.16 and 3.17 .

\section{Acknowledgements}

The authors wish to acknowledge the support of Mulungushi University and the refereed authors for their helpful work towards this paper. They are also grateful to the anonymous peer-reviewers for their valuable comments and suggestions towards the improvement of the original manuscript.

\section{Conflicts of Interest}

Regarding the publication of this paper, the authors declare that, there is no con- 
flict of interest.

\section{References}

[1] Crossley, S. and Hildebrand, S. (1972) Semi-Topological Properties. Fundamenta Mathematicae, 74, 233-254. https://doi.org/10.4064/fm-74-3-233-254

[2] Popa, V. (1988) On Characterizations of Irresolute Multifunctions. Journal of the Universityof Kuwait (Science), 15, 21-26.

[3] Levine, N. (1963) Semi-Open Sets and Semi-Continuity in Topological Spaces. The American Mathematical Monthly, 70, 36-41. https://doi.org/10.1080/00029890.1963.11990039

[4] Maheshwari, S.N. and Prasad, R. (1977) Semi-Open Sets and Semi-Continuous Function in Bitopological Spaces. Mathematical Notes, 26, 29-37.

[5] Popa, V. (1975) Multifunctions and Bitopological Spaces. Bulletin Mathématique de la Société des Sciences Mathématiques de la République Socialiste de Roumanie, 19, 147-152.

[6] Maki, H., Rao, K.C. and Gani, A.N. (1999) On Generalizing Semi-Open Sets and Preopen Sets. Pure and Applied Mathematika Sciences, 49, 17-30.

[7] Popa, V. and Noiri, T. (2000) On m-Continuous Functions. Annals of the University Dunarea de Jos of Galati, 18, 31-41.

[8] Bose, S. (1981) Semi Open Sets, Semi Continuity and Semi Open Mappings in Bitopological Spaces. Bulletin of the Calcutta Mathematical Society, 73, 237-246.

[9] Berge, C. (1963) Topological Spaces: Including a Treatment of Multi-Valued Functions, Vector Spaces and Convexity. Oliver and Boyd Ltd., Edinburgh.

[10] Noiri, T. and Popa, V. (2000) On Upper and Lower m-Continuous Multifunctions. Filomat, 14, 73-86.

[11] Matindih, L.K. and Moyo, E. (2021) On $M$-Assiymetric Semi-Open and $M$-Asymmetric Semicontinuous Multifunctions in Bitopological Spaces. Advances in Pure Mathematics, 11, 218-236. https://doi.org/10.4236/apm.2021.114016

[12] Ewert, J. and Lipski, T. (1983) Quasi-Continuous Multivalued Mappings. Mathematica Slovaca, 33, 69-74.

[13] Kelly, J.C. (1963) Bitopological Spaces. Proceedings of the London Mathematical Society, 3, 71-89. https://doi.org/10.1112/plms/s3-13.1.71 